\title{
Desarrollo rural en El Salvador: una deuda política y social
}

Jorge Luis Quintanilla Trigueros

Cáritas El Salvador, Diócesis de Santa Ana

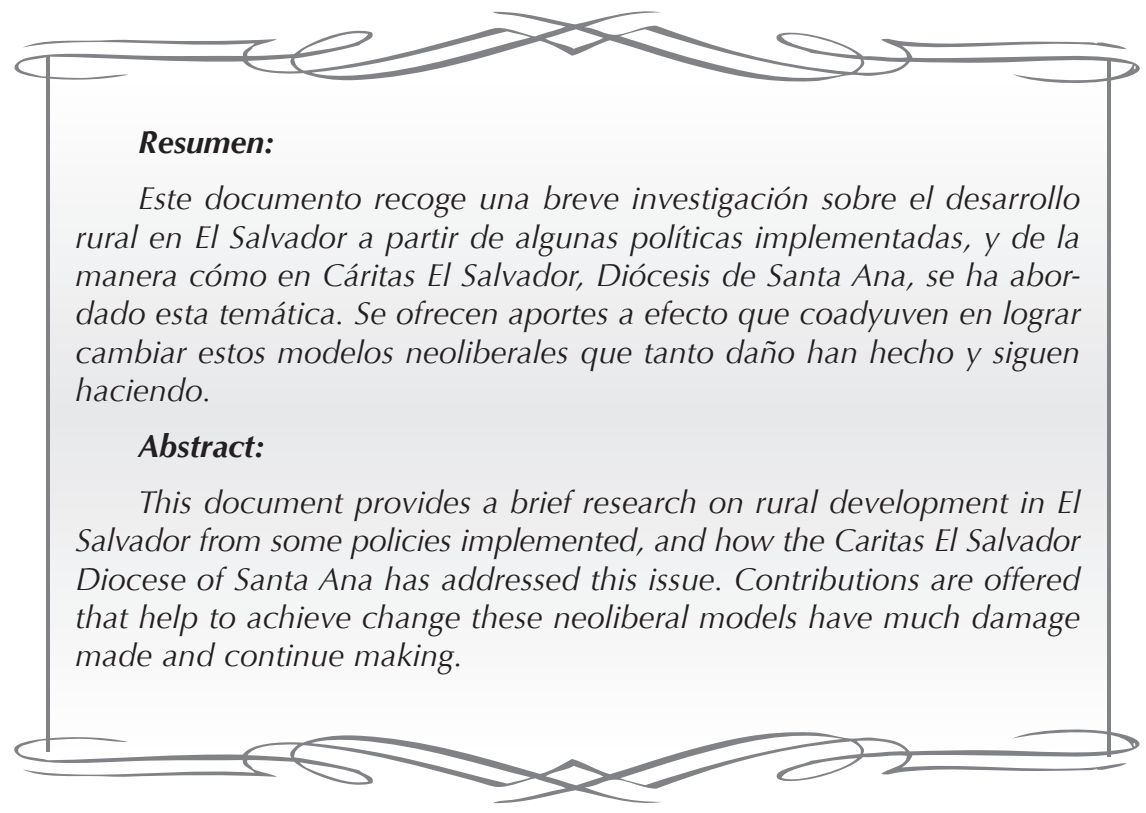

$\mathrm{H}$ ablar de desarrollo es lo que toda la humanidad busca, librar la pobreza por una mejor condición de vida. Desgraciadamente, este concepto se ha ido poco a poco fragmentando $y$ no es lo mismo hablar ahora de desarrollo económico, desarrollo social, desarrollo humano, desarrollo espiritual, desarrollo tecnológico, desarrollo político, incluso muchos países apuestan ahora a un desarroIlo militar. De todo esto surge también la división sobre el desarrollo urbano y el desarrollo rural. A este último se puede ligar la palabra pobreza. Karl Marx, conceptualiza esta palabra: 
La pobreza se vincula con la no satisfacción de necesidades: alimento, vestuario, vivienda, trabajo, etc. Es la realidad cotidiana de un grupo al que se le niega el valor de su trabajo y se le arrebatan las posibilidades de producir para sí.

En la región centroamericana, y en otras partes del mundo también, las consecuencias de la implementación del modelo capitalista es muy cuestionada especialmente por su impacto negativo en los aspectos sociales y ambientales, incluso ahora, por la persistente fragilidad de los mercados de la economía globalizada, efecto que podemos observar en varios países de Europa y Estados Unidos. A ese respecto, la adopción del modelo capitalista en El Salvador condujo a un conjunto de reformas que impusieron nuevas relaciones entre el Estado, el mercado, sector privado y sociedad civil. Es de notar que el paquete de reformas parte de una concepción "economicista": eficiencia económica (resultados versus recursos), definido por gobiernos preocupados esencialmente por una política macroeconómica, esperando que la "mano invisible" del mercado haga el resto. Este período se caracteriza por la predominancia de los equipos económicos. Quedando lo social en un segundo plano.

A pesar del apoyo internacional y de la intención del gobierno de reformar el Estado hacia una economía de mercado, los resultados fueron a medias: impregnados de desviaciones, avances, retrocesos, contradicciones y reformas inconclusas. Esto también ha sucedido en la mayoría de los países de la región. La razón estriba en que no es posible avanzar en ninguna reforma sustantiva (cualquiera que sea) sin apoyo de la opinión pública y, peor aún, en contra de ella. Por lo cual, hablar de desarrollo en Latinoamérica, es hablar de una deuda, y El Salvador no es la excepción, principalmente para las zonas rurales, zona que en estos países ha quedado olvidada en materia de desarrollo social.

En octubre de 1994, a solicitud del gobierno y con el apoyo del Programa de las Naciones Unidas para el Desarrollo (PNUD), se preparó un documento de cooperación técnica para el fortalecimiento institucional de Casa Presidencial. Ahí se dice... "un país en proceso de modernización que debe afrontar reformas económico-sociales requiere:

1. Un diseño estratégico que adquiera la naturaleza de política de Estado. (Consolidación del Estado de derecho, Modernización y Equidad social).

2. Una autoridad política fuerte, que dé liderazgo al gobierno y asegure gobernabilidad. 
3. Una autoridad financiera fuerte que garantice reglas del juego estables y no sometida a cambios arbitrarios y sorpresivos.

4. Un proceso razonable de descentralización que tenga en cuenta las dimensiones y condiciones especiales del país.

En 1997 se crea por decreto presidencial el Consejo Nacional para el Desarrollo Sostenible (CNDS). En el mismo año, y siempre a instancia de la Presidencia de la República, se conformó la Comisión Nacional de Desarrollo, integrada por un grupo reducido de profesionales o empresarios, cuyo propósito era entregar al país las bases para el plan de nación. Con el documento se daba inicio a un proceso de análisis, discusión y acuerdos básicos entre las principales fuerzas políticas, sociales y económicas del país que, en su fase final, daría como resultado el Acuerdo Nacional del plan de nación. Así, la CND no fue un organismo ejecutor sino de consulta que promueve un enfoque territorial, algo que era nuevo para ese tiempo.

Pero, paradójicamente, la composición del presupuesto para esos años revela que uno de los mayores vacíos de los programas de gobierno se encuentra en el área del fomento del desarrollo social. Para ese año, la población no encuentra ni las ganas ni los motivos para movilizarse en repudio ni a favor de nada. La apatía y el desinterés por la cuestión pública, por el todo social, ha penetrado tan hondo en la población. Esto no es gratuito. Mucha de la responsabilidad recae en los partidos políticos y su falta de figuras líderes, capaces y creíbles. Además, el destape de los múltiples escándalos financieros y la parcializada modalidad en que los medios de comunicación cubrieron muchos de los más importantes hechos políticos de ese año, son variables que han de tenerse en cuenta para explicar el desvanecimiento de la sociedad civil.

Solo falta ver lo que el Programa de las Naciones Unidas para el Desarrollo (PNUD) sugirió al Estado salvadoreño. La necesidad imperativa de adoptar un nuevo modelo de desarrollo, el cual tenga una mayor visión hacia lo social no dependiente de la situación económica; lo cual contrasta con lo que los gobiernos de este país han venido definiendo históricamente, los cuales nunca pusieron a la población como fin último y se centraron en el crecimiento económico.

Sabiendo muy bien todos que la razón de ser de un Estado es procurar el beneficio de su población, El Salvador durante los últimos 60 años reprodujo pobreza y desigualdad entre la población salvadoreña, lo que provocó que las políticas sociales de los gobiernos salvadoreños no tuvieran un rol protagónico en los modelos de desarrollo eco- 
nómico. "En El Salvador del último siglo, la política social ha sido más bien una variable dependiente y no independiente de los resultados de la política económica".

Luego de recordar la realidad política en la cual se ha ido desarrollando nuestro país en el último siglo, es necesario centrarse en definir qué entender cuando nos referimos al desarrollo rural. La mayoría piensa que vivir en el área rural supone la ausencia o deficiencias de muchos de los servicios de los que se gozan con normalidad en las zonas urbanas (servicios básicos y otros como: salud, nivel educativo alto, precios de los productos más bajos, oportunidades de empleo, centros comerciales, etc.). Muchos de nosotros incluso nos asustaríamos cuando nuestro celular en la pantalla dice "sin servicio" o no hay acceso a internet.

A estos problemas tradicionales del ámbito rural, se suman los efectos negativos de otros nuevos, que afectan y profundizan la situación de pobreza y exclusión ya existente. Por ejemplo, los problemas ambientales relacionados con la deforestación, la degradación de suelos y el acceso al agua; sistemas productivos muy dependientes de insumos externos que tienden a encarecerse; marcadas desigualdades en el acceso a la tierra, la tecnología y el conocimiento; debilidades políticoinstitucionales; y un casi total alejamiento de las nuevas dinámicas socioculturales relacionadas con el internet. Esta es la realidad con la cual se presenta la población rural y que la ponen en franca desventaja con respecto a la población urbana.

Pero realmente hemos olvidado incluir el punto de vista de las personas que viven en esta zona, las cuales se las ingenian para sobrevivir día a día. Y lo más notable es que si regresamos muchos años atrás, éstas no manejaban el término de pobreza, como el que ahora los señala en un mapa fabricado por un gobierno anterior para definir a todo nuestro país como una calamidad total, ya que nos define a todos en pobreza a la cual le da diferentes niveles. Este mapa fue y es una carta de presentación, ya que esta imagen nos muestra como necesitados de recibir un apoyo económico extranjero. Pero de no ser así, el país y sus habitantes estarían destinados a sufrir una situación de decadencia.

Al analizar los muchos años de trabajo, en Centroamérica los resultados de los proyectos de desarrollo rural han sido muy insignificantes, cuando no nulos: las zonas rurales siguen siendo pobres y siguen estando excluidas. Este fracaso se ha debido fundamentalmente a que la inversión estatal y de Ong's en la zonas rurales ha sido siempre marginal, pero principalmente a la falta de participación de la población en la definición de los proyectos de desarrollo y en la ejecución de los mismos, a una visión reduccionista y segmentada de la realidad y a la 
descoordinación entre las instancias que han trabajado en los territorios.

A pesar de los avances en las reformas políticas, es necesario consolidar y profundizar la democracia y, a la vez, darle eficiencia al aparato público. En este sentido, las reformas son inconclusas o parciales. El documento Bases para el Plan de Nación insiste en avanzar en las reformas al sistema electoral y a ley de partidos políticos. Asimismo, plantea la necesidad de reformas en el reordenamiento político administrativo, la redefinición de roles de gobierno (local y nacional), la garantía de la moralidad pública (corrupción), fundamentales para un servicio público eficiente. En cuanto a las reformas económicas y sociales, los resultados no se perciben o son contrarios a lo esperado, sea porque requieren más tiempo o el modelo aplicado no responde.

Partiendo de este panorama, Caritas El Salvador, Diócesis de Santa Ana, ante esta necesidad de enfrentar el reto del desarrollo rural ha venido comprendiendo que es necesario acompañar un trabajo de manera conjunta, con visión de formar parte de una estrategia que vaya de lo local a lo regional y a un desarrollo de territorio, la cual compartimos en gran parte con la planteada por el SICA, conocida como ECADERT. Se trata de una iniciativa que permitirá que unos aprendan de los otros a medida que se vayan obteniendo resultados.
Estudiando la estrategia, "ECADERT que plantea adoptar un modelo de desarrollo solidario y efectivamente incluyente, cuyo sustento sea una sociedad rural organizada y un tejido social fortalecido, capaz de incorporar a todas las fuerzas sociales del medio rural en carácter de autoridades locales, ciudadanos, productores y consumidores responsables. Se busca transformar la dinámica del territorio mediante transformaciones simultaneas en el ámbito político-institucional, sociocultural, económico-productivo, y ambiental, de conformidad con el potencial propio de cada territorio". Todo ello con el fin de alcanzar equidad social y cultural, gobernabilidad política, competitividad económica y sostenibilidad.

Es muy acertado que la implementación del modelo ECADERT inicie con un proceso de formación para quienes serán los gestores de esta nueva visión sobre el desarroIlo rural territorial. La cual no debe estar basada en instituciones o técnicos externos al área rural. Por lo que es necesario iniciar una página diferente en la cual las personas del área rural sean verdaderamente las guías que puedan llevar y desarrollar esta iniciativa y evitar seguir haciendo más de lo mismo.

Desafortunadamente esta idea aún no se lleva acabo; falta que se haga efectivo el compromiso de los gobiernos y de las distintas organizaciones que colaborarán en el 
desarrollo de los primeros territorios rurales elegidos para iniciar en ellos la nueva estrategia ECADERT.

Mientras tanto, Cáritas a través de sus proyectos ha comenzado con la gente a darle forma a esta visión de fortalecimiento de los territorios, pues es importante exigir dar este paso que implica una renuncia al protagonismo politiquero de los gobiernos, que siempre han impulsado programas de desarrollo diseñados para poder someter voluntades de votantes. Ahora está el reto de poner a la disposición de las comunidades los programas de desarrollo y los recursos necesarios para llevarlos adelante, y así se honre la deuda que el país y la región tienen con el mundo rural.

Un punto importante del cual partimos es despertar la conciencia colectiva en las comunidades, promoviendo la formación de grupos organizados por comunitarios que sean capaces de identificar las principales necesidades que les aquejan y poner en marcha un proceso de aprendizaje. Cáritas ha identificado este proceso de aprendizaje como formación de promotores/as voluntarios/as, las cuales son personas con diferentes cualidades y habilidades que a través de una curricula comienzan un proceso de formación en el que van descubriendo y puliendo sus capacidades para ser verdaderos líderes propositivos para sus comunidades. El objetivo es que se pueda dar el siguiente paso en la formación de una estructura que los agrupe en comunidades con mismos objetivos (una intercomunal) en la cual puedan compartir sus experiencias de aprendizaje y tomar nuevas, que irán dando capacidades para ver y entender mejor las problemáticas de sus comunidades y buscar en conjunto posibles alternativas. El fin último del proceso es formar una conciencia colectiva y al mismo tiempo promover la capacidad de dirección y liderazgo, procurando la inclusión de los diferentes actores del desarrollo local de sus propios territorios (centro escolar, unidad de Salud, iglesias, alcaldía, gobernación, protección civil, policía, Ong's, etc.). De lo que se trata es que todas estas instancias puedan construir estrategias que faciliten el desarrollo social, económico y político de las comunidades o región.

El desarrollar proyectos con objetivos específicos para nosotros es una oportunidad para poder acompañar a las personas del área rural a descubrirse como verdaderos actores capaces de cambiar e incidir en los procesos de las políticas sociales, llevando a las personas como eje principal del desarrollo económico local, regional de territorio y del país, y que éstas tengan un enfoque de derechos humanos. Para esto, la fórmula debería ser sencilla: a mayor inversión del Estado en su población aumentaría el nivel de bienestar social, obteniendo mejores resultados económicos. Para ello, el Estado tendría que corregir 
los desbalances macroeconómicos nacionales, aumentar los niveles de ahorro, la inversión y equilibrar las finanzas públicas, buscando financiar las políticas sociales con recursos propios.

Esta es una apuesta que debemos exigir al gobierno que haga en la personas, lo cual conlleva una gran responsabilidad para el gobierno, pero aún más para la población civil que debe de apoyar y fomentar estas iniciativas tanto en el área rural como urbana.

Vale la pena analizar que el gobierno actual ha anunciado que la inversión en el área social alcanzaría los 818 millones de dólares en 2011, un aumento del $70 \%$ con respecto a 2010. "Nunca como ahora la salud, la vivienda, la educación, la alimentación, y toda las necesidades básicas de las grandes mayorías habían recibido la atención que hoy se brinda con el dinero público. Nuestro modelo económico debe ser la apuesta por el pueblo salvadoreño, por la mejora de sus capacidades, por la producción nacional, por la recuperación del campo y por la competi- tividad de nuestros emprendedores", dijo el presidente Mauricio Funes, durante la presentación del estudio.

Si analizamos su estrategia nos daremos cuenta que "El crecimiento económico estaría fundamentado en el fortalecimiento de la relación ahorro-inversión y en el aumento de la productividad laboral". La estrategia consiste en la construcción de un círculo virtuoso que eleve los niveles de ahorro, inversión, competitividad, crecimiento y empleo, permitiendo una mejora en la macroeconomía nacional y aumentar el nivel de bienestar de la gente.

Investigadores del PNUD analizaron los modelos económicos -agroexportador, industrialización, aumento de exportaciones y atracción de inversiones- aplicados en los últimos 70 años y concluyeron que no tomaron en cuenta a la gente, usando los bajos salarios como la principal ventaja para insertar al país en los mercados internacionales y haciendo del subempleo la forma predominante de sobrevivencia de la mayoría de la población en la actualidad.

Un vistazo a la implementación de los modelos económicos en el país confirmaría que, hasta ahora, todos los caminos intentados nos han llevado al mismo lugar. No colocar a las personas en el centro de las políticas ha sido, sin lugar a dudas, el principal error de los sucesivos experimentos económicos de El Salvador.

A pesar de que los modelos económicos generaron importantes avances en materia de desarrollo para el país, el informe hace hinca- 
pié en que El Salvador ha crecido "a un ritmo ligeramente inferior al de los otros países de desarrollo humano medio, por lo que su posición ha permanecido casi inalterada dentro de la clasificación que construye anualmente el PNUD".

En la actualidad, El Salvador, con un índice de desarrollo humano (IDH) de 0.659, se ubica en la posición 90 de la lista de 162 países estudiados por el informe anual del PNUD. El índice lo ubica como un país de desarrollo humano medio. "Sin embargo, si se tomara en cuenta la desigualdad existente dentro del país, una de las mayores de América Latina, El Salvador experimentaría una caída en 14 puntos, colocándose en un nivel de desarrollo humano similar al de Honduras, ubicado en la posición 116 de la lista», dice el documento.

Dentro de El Salvador, dice el estudio, se mantienen brechas de desigualdad importantes: "Los habitantes de Antiguo Cuscatlán disfrutan de un IDH similar al de un país como Portugal, mientras municipios como Jutiapa (Cabañas) y Lislique (La Unión) se encuentran al nivel de los empobrecidos países del África subsahariana".

Un Estado frágil o una sociedad civil débil no pueden lograr encauzar al país por la senda del desarrollo sostenible. En este sentido, las reformas deben orientarse a fortalecerlos a ambos. El Salvador tiene que realmente "abrirse hacia adentro" para luego "abrirse hacia afuera".

Independientemente de si se está de acuerdo o no con los contenidos de los documentos o los mecanismos utilizados, no cabe duda de que para el país es altamente positivo (y llamativo) la conformación de grupos de análisis, discusión o reflexión para incitar intereses y posiciones sobre la visión o imagen futura de la nación, partiendo de una sociedad desintegrada por la pobreza y exclusión social.

Eficiencia: al Estado en sus nuevos roles y funciones. Este planteamiento, desde la perspectiva de la administración pública, pretende dar mayor transparencia, eficacia y eficiencia al proceso de toma de decisiones del Estado. Comenzando desde lo local hasta las instituciones de mayor jerarquía del gobierno. Desarrollo económico local: Este enfoque debe ser complementado con la profundización y ampliación del proceso de integración de las comunidades, municipalidades etc.

La descentralización es un proceso que no sólo apunta a la eficiencia de los servicios públicos, sino también a la consolidación de la democracia política, económica y social participativa. Por otro lado, no puede lograrse eficiencia haciendo «piñatas» de las instituciones públicas, como «botín» del ganador de las elecciones y sus aliados. Esta 
forma de corrupción atenta contra el rendimiento y espíritu del servicio público.

Como decía Eric Calcagno: "se afirma que debemos subirnos al tren de la modernidad (como si sólo hubiera uno), aunque no sepamos si va donde queremos ir, e ignoremos si nos van a subir como pasajeros o como personal de servicio al que se devuelve al punto inicial una vez terminado el viaje, o si al llegar al punto del destino seremos trabajadores inmigrados. Es decir, nos aconsejan que como países adoptemos una conducta que ningún liberal (y tampoco una persona cuerda) seguiría en una estación de ferrocarril" (Calcagno, 1995, Argentina, quien es un crítico del modelo Neoliberal).

Sin lugar a dudas, Centroamérica y El Salvador optimizarán los beneficios de insertarse al proceso mundial aperturista. Ambos, integración y desarrollo económico local, resultan inevitables a largo plazo para lograr y mantener un proceso de desarrollo sostenible.

En este sentido, no se debe perder de vista el objetivo de insertarse (con la apertura) al proceso globalizante: conducir a los países de la región a un desarrollo económico, social, político, cultural, sustentable ambientalmente y sostenible en el tiempo; vale decir, que garantice una mejor calidad de vida a las futuras generaciones.
La educación, la salud, el acceso a la vivienda, a los servicios básicos, la seguridad ciudadana, la práctica y confianza en la aplicación absoluta de la ley, la protección del medio ambiente vinculado a calidad de vida y sostenibilidad, son elementos que contribuyen a engendrar verdaderos emprendedores (empresarios creadores de riqueza, no rentistas), con confianza en su capacidad individual y seguros de que sus iniciativas serán aceptadas por la sociedad

Como conclusión de estas ideas sobre el desarrollo sostenible del área rural, sostenemos: Nada mejor para orientar las reformas del Estado que un estilo de desarrollo integral e integrador de la sociedad (actual y futura), que respete las minorías y ponga como centro del desarrollo al ser humano. Un paradigma incompatible con crecientes niveles de pobreza, exclusión y desintegración social.

Un nuevo estilo de desarrollo sostenible en el acceso y uso de los recursos naturales y en la preservación de la biodiversidad; socialmente sostenible, en la reducción de la pobreza y desigualdades sociales y promueva la justicia y equidad; culturalmente sostenible, en la preservación de valores y prácticas; y políticamente sostenible, que profundice la democracia y promueve la participación ciudadana en la toma de decisiones públicas, y con ello dé gobernabilidad al sistema. 
Este nuevo estilo de desarrollo requiere una nueva ética del crecimiento, una ética en la cual se dobleguen los objetivos económicos a las leyes de funcionamiento de los sistemas sociales, al respeto de la dignidad humana y de mejoría de la calidad de vida de las personas.

A diferencia de las reformas recientes impulsadas desde la perspectiva ortodoxa del mercado, esta "nueva" visión del desarrollo tiene un horizonte a mediano y largo plazo. Por ello es necesaria la intervención eficiente del Estado: fuerte pero limitado. Es un Estado que acompaña (y promueve) a la sociedad civil, igualmente fuerte, organizada y participativa en la toma de decisiones sobre cuestiones que atañen a intereses relevantes de la sociedad y donde la mano invisible del mercado no funciona. El nuevo Estado debe ser más gobernable. Como se dijo, la sostenibilidad del sistema político democrático es sinónimo de gobernabilidad.

A este respecto, es clave que los gobernantes (a todo nivel) no sólo representen electores sino también sean instrumento de participación de las comunidades. Esta sociedad debe sentir que sus gobernantes la representan y trascienden el interés partidario en pro del beneficio colectivo, vale decir, hacen políticas de Estado. En última instancia, ello depende de la capacidad de la sociedad para definir políticas de Estado, verda- dera condición fundamental de una genuina y duradera convivencia democrática.

En este sentido, el Estado debe impulsar reformas en los mecanismos de representatividad política, en el logro de un sistema electoral transparente e independiente, en la democratización y financiamiento transparente de los partidos políticos, y alcanzar una gestión pública ejercida con sentido de servicio público (participación, credibilidad, moralidad y eficiencia, que son pilares de la gobernabilidad).

Este Estado debe ser descentralizado. Este proceso abona a la sostenibilidad política-gobernabilidad del sistema vía eficiencia y transparencia en la toma de decisiones, a través de la supervisión y participación ciudadana; acerca gobernantes y gobernados. En cuanto a la sostenibilidad de las otras áreas del desarrollo integral (económico, social, ambiental y cultural), la descentralización es estratégica en la promoción del desarrollo económico local. Esta reforma estratégica pasa por el reordenamiento políticoadministrativo del territorio, lo cual demanda un sólido compromiso nacional, por lo profundo y complejo del tema.

Un nuevo Estado también debe ser estratégico. La Presidencia debe dar el ejemplo de moralidad, eficiencia, consistencia y confianza pública en sus decisiones. 
El Estado debe promover el enfoque del desarrollo económico local. No basta la política o equilibrios macroeconómicos o políticas sectoriales, se requiere simultánea y complementariamente desarrollar la institucionalidad. El diseño y fortalecimiento del sistema nacional para el desarrollo sostenible significa un fuerte impulso institucional en esta dirección. Ello porque facilita Ilevar al nivel micro o meso económico en el territorio el apoyo sectorial o el acceso a universidades, centros de investigación, mercados, etc. Asimismo, el fortalecimiento del tejido social en la comunidad contribuirá a facilitar el cumplimiento de los requisitos ambientales en las empresas y actividades desarrolladas en las mismas.

El Estado debe promover el financiamiento interno del desarrollo. Hay que fomentar el ahorro interno (inversión) que financie el desarrollo de actividades productivas sostenibles a largo plazo; el Estado debe hacer un esfuerzo muy grande para darle sostenibilidad a la productividad.

El Estado debe asumir responsablemente la sustentabilidad ambiental, como condición indiscutible y prioritaria que garantice la calidad de vida a las nuevas generaciones, dado el nivel de deterioro ambiental en El Salvador (segundo lugar en desertificación, bajo Haití).
El Estado deberá ser el garante de la integración social. Al inicio de este artículo se pone a la erradicación de la pobreza como el objetivo de la transformación del Estado y la sociedad civil. Precisamente, el proceso de modernización y apertura en El Salvador no es sostenible, pues lo social es marginal respecto a lo económico. Diría que ni siquiera ha existido una política social en los términos que dice Carlos Blanco: "La política social no es, muy a menudo, sino la ambulancia que recoge los cadáveres dejados por la política económica". La equidad es la sostenibilidad en la variable social, por tanto, es fundamental a largo plazo también como factor de competitividad y productividad del país.

Para finalizar, después de una década de reformas del Estado, con la concepción "mercadeana" de achicarlo, de tratar de limitarlo, ojalá a las funciones tradicionales de defensa, seguridad y justicia (éstas las "mercadean" los libertarios), se concluye:

1. Que el Estado "privatizado" no funcionó ni siquiera para desarrollar o regular los mercados, menos para combatir la pobreza, la exclusión social o la destrucción ambiental.

2. Es urgente fortalecer el Estado, darle dirección de largo plazo 
y eficiencia en la acción para que acompañe a la sociedad civil en el combate contra la pobreza, como un inicio para mejor la calidad de vida de los salvadoreños (sostenidamente).

3. El Estado es insustituible para abordar los intereses de la nación a mediano y largo plazo (necesidades sociales, ambiente, seguridad, etc.).
Es decir, el desarrollo sostenible tanto para el área rural como urbana demanda: Un Estado fuerte, limitado, eficiente y democrático, y una sociedad civil igualmente fuerte, organizada y participativa. De lograrse, no cabría duda de que el siglo XXI sería de esperanza y cambios en la vida de nuestra sociedad urbana como rural. 